PROCEEDINGS OF THE

AMERICAN MATHEMATICAL SOCIETY

Volume 138, Number 8, August 2010, Pages 2815-2826

S 0002-9939(10)10286-X

Article electronically published on April 14, 2010

\title{
SENSITIVE DEPENDENCE ON INITIAL CONDITIONS AND CHAOTIC GROUP ACTIONS
}

\author{
FABRIZIO POLO
}

(Communicated by Bryna Kra)

\begin{abstract}
A continuous action of a group $G$ on a compact metric space has sensitive dependence on initial conditions if there is a number $\varepsilon>0$ such that for any open set $U$ we can find $g \in G$ such that $g . U$ has diameter greater than $\varepsilon$. We prove that if a countable $G$ acts transitively on a compact metric space, preserving a probability measure of full support, then the system either is minimal and equicontinuous or has sensitive dependence on initial conditions. Assuming ergodicity, we get the same conclusion without countability. These theorems extend the invertible case of a theorem of Glasner and Weiss. We prove that when a finitely generated, solvable group acts transitively and certain cyclic subactions have dense sets of minimal points, the system has sensitive dependence on initial conditions. Additionally, we show how to construct examples of non-compact monothetic groups and transitive, non-minimal, almost equicontinuous, recurrent $G$-actions.
\end{abstract}

\section{INTRODUCTION}

By a topological dynamical system we shall mean a continuous action of a topological group $G$ on a compact metric space $(X, d)$, i.e., a continuous map $\pi: G \times X \rightarrow$ $X:(g, x) \mapsto g . x$ with the property that $g .(h . x)=g h . x$ and $e . x=x$ where $e$ is the identity element of $G$. We denote such a system by the pair $(X, G)$. If $T: X \rightarrow X$ is a homeomorphism, we may also call $(X, T)$ a topological dynamical system. In this case we are referring to the obvious action $\mathbb{Z}$. Occasionally we refer to theorems in which $T$ is not assumed to be invertible. It will be made clear when this is the case.

To understand the following property of dynamical systems is the primary goal of this paper:

Definition 1.1. A topological dynamical system $(X, G)$ is said to have sensitive dependence on initial conditions (or is said to be sensitive) if there is some $\epsilon>0$ such that for all $x \in X$ and for every open neighborhood $U$ of $x$ there exists $y \in U$ and $g \in G$ such that $d(g . x, g . y)>\epsilon$.

It is worth noting that one need not (overtly) mention points in the definition of sensitive dependence on initial conditions; see the following equivalent definition: there exists $\varepsilon>0$ such that for all open sets $U, \sup _{g \in G} \operatorname{diam}(g . U)>\varepsilon$.

Received by the editors July 14, 2009 and, in revised form, November 12, 2009 and November 13, 2009.

2010 Mathematics Subject Classification. Primary 28D05, 28D15, 37A05, 37B05; Secondary 22B99.

(c) 2010 American Mathematical Society 2815

Reverts to public domain 28 years from publication 
Definition 1.2. A point $x \in X$ is called an equicontinuous point if for all $\varepsilon>0$ there exists $\delta>0$ such that for any $y \in X, d(x, y)<\delta$ implies $d(g . x, g . y)<\varepsilon$ for all $g \in G$. A point which is not an equicontinuous point will be called a sensitive point.

A system $(X, G)$ is said to be equicontinuous if the set of all maps $\{x \mapsto g . x: g \in$ $G\}$ is an equicontinuous family. If $(X, G)$ is equicontinuous, then it is easily seen that every point is equicontinuous. Conversely, if every point is equicontinuous, then for each $\varepsilon>0$ and each point $x$ there exists $\delta_{x}>0$ such that $g \cdot B_{\delta_{x}}(x)$ has diameter less than $\varepsilon$ for all $g \in G$. From the balls $B_{\delta_{x}}(x)$ we can choose a finite subcover $\mathcal{U}$. Let $\delta$ be a Lebesgue covering number for $\mathcal{U}$. Then, if $d(x, y)<\delta$, there is some $U \in \mathcal{U}$ containing $x$ and $y$. So, $d(g . x, g . y) \leq \operatorname{diam}(g . U)<\varepsilon$ for all $g$. This proves that every point is equicontinuous if and only if the system is equicontinuous.

Now we define a new metric $d_{\infty}$ on $X$ by $d_{\infty}(x, y)=\sup _{g \in g} d(g . x, g . y)$. Consider the identity map Id $:(X, d) \rightarrow\left(X, d_{\infty}\right)$. When $G$ is a monoid, $\mathrm{Id}^{-1}$ is a contraction and hence continuous. If $(X, G)$ is equicontinuous, it is easy to see that Id is a homeomorphism. Also, if $\left(X, d_{\infty}\right)$ is compact, then Id has compact domain and Hausdorff range and so is a homeomorphism. A sensitive point is precisely a discontinuity point for Id.

Logically speaking, the assertion that a system has sensitive dependence on initial conditions is stronger than the assertion that every point is sensitive (they differ in the order of quantifiers). But, by a category argument (see the excellent article [1]), one can prove the following:

Proposition 1.3. A transitive system is sensitive at each point if and only if it has sensitive dependence on initial conditions.

Definition 1.4. A system is called almost equicontinuous if it has a dense set of equicontinuous points.

It follows from Corollary 2 in 3 that for a single transitive map, either the system is sensitive, or the set of equicontinuous points is exactly the same as the set of transitive points. This is commonly known as the Auslander-Yorke dichotomy theorem. It holds in the non-invertible case as well.

The following standard definitions are discussed in much greater detail elsewhere. For instance, [11] and [6] are good references. From now on we take $G$ to be a group. We say $(X, G)$ is topologically transitive (or transitive for short) if for any open $U$ and $V$ there exists $g \in G$ such that $U \cap g . V \neq \emptyset$. In our setting, this is equivalent to the existence of a dense set of points $x_{0} \in X$ such that $G$. $x_{0}$ is dense in $X$ (see Proposition 3.2 from [9]).

A system is called minimal if every point has dense orbit. A point will be called minimal if its orbit closure is a minimal system. When the acting group is discrete, a point $p \in X$ is called a periodic point if $G . p$ is finite. Periodic points are easily seen to be minimal. Saying that $x$ is a minimal point is equivalent to requiring that for every open neighborhood $U$ of $x, R:=\{g \in G: g \cdot x \in U\}$ be left syndetic; that is, there is a finite subset $F$ of $G$ such that $F R=G$.

In a group, any set which contains an infinite set of the form $S S^{-1}=\left\{s t^{-1}: s, t \in\right.$ $S\}$ is called a $\Delta$ set. A set is called $\Delta^{\star}$ if it intersects every $\Delta$ set. A $\Delta^{\star}$ set which is symmetric (i.e., equal to its inverse) is always syndetic. To see this, suppose the set $L \subset G$ is symmetric but not syndetic. Let $g_{0}=1_{G}$ and assume we have already chosen distinct elements $g_{0}, \ldots, g_{n-1}$ with the property that $g_{i} g_{j}^{-1} \notin L$ when $i \neq j$. 
Since $L$ is not syndetic we can choose $g_{n}$ such that $\left\{g_{n} g_{0}^{-1}, \ldots, g_{n} g_{n-1}^{-1}\right\}$ misses $L$. Since $L$ is symmetric we also know $\left\{g_{0} g_{n}^{-1}, \ldots, g_{n-1} g_{n}^{-1}\right\}$ misses $L$. Inductively, we see that $L \cap S S^{-1}=\emptyset$ where $S=\left\{g_{n}\right\}_{n}$; that is, $L$ is not $\Delta^{\star}$.

A probability measure $\mu$ on the Borel $\sigma$-algebra $\mathcal{B}=\mathcal{B}(X)$ is invariant if $\mu(A)=\mu\left(g^{-1}\right.$.A) for all $A \in \mathcal{B}, g \in G$. All measures should be assumed to be Borel measures. The measure $\mu$ is said to be ergodic if any invariant set has measure one or zero.

In the literature on sensitivity, many results have a common theme: under some additional hypothesis, an almost equicontinuous system must be equicontinuous. This gives a dichotomy: any system satisfying the additional hypothesis is either equicontinuous or sensitive. Topological hypotheses are popular (see [4], 1], and [7]). Measure theoretic hypotheses also appear (see [7]). The next two theorems are particularly nice examples.

Theorem 1.5 (Glasner and Weiss []). If $(X, T)$ is a transitive topological system equipped with an invariant probability measure $\mu$ of full support, then either $(X, T)$ has sensitive dependence on initial conditions or it is minimal and equicontinuous.

Theorem 1.6 (Akin, Auslander, and Berg [1]). Let $(X, T)$ be a (possibly noninvertible) transitive system. If the set of all minimal points is dense, then either the system has sensitive dependence on initial conditions or $X$ is a minimal equicontinuous system.

Both of these theorems are generalizations of their predecessor in [4]:

Theorem 1.7 (Banks, Brooks, Cairns, Davis, and Stacey [4]). Let $(X, T)$ be a (possibly non-invertible) transitive system. If the set of periodic points is dense, then either the system has sensitive dependence on initial conditions or $X$ is a finite set.

A periodic point is a minimal point, so Theorem 1.6 implies Theorem 1.7. Theorem 1.5 implies Theorem 1.7 because the existence of a dense set of periodic points allows one to easily construct an invariant measure of full support by adding weighted counting measures on periodic orbits.

In this paper we derive similar results for the actions of more general groups. Our main results are Theorems [1.8, 1.9, and 1.12, Theorem 1.8 generalizes the invertible case of Theorem 1.5 .

Theorem 1.8. Let $(X, G)$ be a transitive system with $G$ countable. If $X$ admits an invariant probability measure of full support, then the system is either minimal and equicontinuous or sensitive.

Theorem 1.9. Let $(X, G)$ be a transitive system. If $X$ admits an ergodic invariant probability measure of full support, then the system is either minimal and equicontinuous or sensitive.

A close examination of the proof in [4] of Theorem 1.7 reveals that (with obvious modifications) it already works for any group action. However, Theorem 2.5 from [1] is more general so we include it here for completeness.

Theorem 1.10. Let $G$ be a group and let $(X, G)$ be a transitive system having a dense set of minimal points. Then, either $(X, G)$ has sensitive dependence on initial conditions or $X$ is minimal and equicontinuous. 
Heuristically speaking, when $G$ is a large group the requirement that the action have dense periodic or minimal points is quite strong. Does a result like Theorem 1.10 hold if we require dense minimal points only for certain subactions? Indeed, when $G$ is solvable there is such a theorem. In order to state it we need another definition.

Definition 1.11. If a finite set $S$ generates a solvable group $G$ we will say $S$ is nice if $G^{(n)}$ is generated by $S \cap G^{(n)}$ (where $G=G^{(0)}$ and $G^{(n+1)}=\left[G^{(n)}, G^{(n)}\right]$ ).

Theorem 1.12. Let $S$ be a nice generating set for a finitely generated solvable group $G$ which acts transitively on a compact metric space $X$. Given $s \in S$, write $\langle s\rangle$ for the subgroup generated by $s$. If the system $(X,\langle s\rangle)$ has a dense set of minimal points for each $s$, then $(X, G)$ either is minimal and equicontinuous or has sensitive dependence on initial conditions.

\section{Almost equicontinuity And transitivity}

In this section we demonstrate the existence of many transitive, almost equicontinuous systems which are not minimal and equicontinuous. We prove analogues of Theorems 4.2 and 4.6 from [2]. The dichotomy type theorems discussed in the introduction use additional hypotheses to eliminate the possibility of almost equicontinuous but not equicontinuous systems. Without the knowledge that such examples exist, the strength of this kind of theorem is questionable.

Lemma 2.1. Let $(X, G)$ be a topological dynamical system with an equicontinuous point $x$. If $y \in X, g_{n} \in G$ and $g_{n} . y \rightarrow x$, then $y$ is also equicontinuous and has the same orbit closure as $x$.

Proof. Given $\varepsilon>0$ there is a neighborhood $U$ of $x$ such that $\operatorname{diam}(g \cdot U)<\varepsilon$ for all $g \in G$. Fix $h$ such that $h . y \in U$. Then $V:=h^{-1} . U$ is a neighborhood of $y$ with $\operatorname{diam}(g . V)<\varepsilon$ for all $g \in G$. Thus $y$ is an equicontinuous point. Since $d\left(g \cdot x, g h^{-1} . y\right)<\varepsilon$ for all $g \in G$, we see that the orbit of $x$ is $\varepsilon$-dense in the orbit of $y$ and the orbit of $y$ is $\varepsilon$-dense in the orbit of $x$. Thus, taking closures of each orbit yields equal sets.

Proposition 2.2. Let $(X, G)$ be a system with a transitive point $x$. The following are equivalent:

(1) $(X, G)$ is almost equicontinuous.

(2) $x$ is an equicontinuous point.

(3) For all $\varepsilon>0$ there exists $\delta>0$ such that for all $g, h \in G, d(h . x, x)<\delta$ implies $d($ gh. $x, g . x)<\varepsilon$.

(4) $d$ and $d_{\infty}$ induce the same topology on the set of transitive points.

Proof. Assume (2). Then any translate of $x$ is an equicontinuous point, and (1) follows. Now we prove that (3) implies (2). Fix $\varepsilon>0$ and choose $\delta>0$ so that $d(h . x, x)<\delta$ implies $d(g h . x, g . x)<\varepsilon / 2$ for all $g \in G$. Now fix $g \in G$ and suppose $d(x, y)<\delta / 2$. Choose $h$ to make $h . x$ close enough to $y$ that $d(g h . x, g . y)<\varepsilon / 2$ and $d(x, h . x)<\delta$. Then

$$
d(g . x, g . y) \leq d(g \cdot x, g h . x)+d(g h . x, g \cdot y)<\varepsilon / 2+\varepsilon / 2 .
$$

We will now prove that (1) implies (4) and (4) implies (3). For (1) implies (4), it suffices to show that a sequence $x_{n}$ of transitive points converges under $d$ to another transitive point $x$ if and only if the same is true under $d_{\infty}$. One direction 
is obvious. For the other direction, suppose $x_{n} \rightarrow x$ under $d$. By assumption $x$ is transitive and hence equicontinuous by Lemma 2.1. For any $\varepsilon>0$, when $n$ is sufficiently large, we have $d\left(g \cdot x_{n}, g \cdot x\right)<\varepsilon$ for all $g \in G$; that is, $d_{\infty}\left(x_{n}, x\right) \leq \varepsilon$. Therefore $d_{\infty}\left(x_{n}, x\right) \rightarrow 0$, as desired.

To see that (4) implies (3), suppose $h_{n} \in G$ are such that $d\left(h_{n} . x, x\right) \rightarrow 0$. Then $d_{\infty}\left(h_{n} . x, x\right) \rightarrow 0$ as well, whence (3).

Proposition 2.3. Suppose that $G$ acts transitively by isometries on a possibly non-compact metric space $X_{0}$ and $\iota: X_{0} \rightarrow X$ is a uniformly continuous metric $G$-compactification; i.e., $(X, G)$ is a compact metric system and $\iota$ is a uniformly continuous, $G$-equivariant homeomorphic embedding of $X_{0}$ onto a dense subset of $X$. Then $(X, G)$ is an almost equicontinuous, transitive system with $\iota\left(X_{0}\right)$ contained in the transitive points of $X$. Conversely, every almost equicontinuous, transitive system $(X, G)$ arises in this way: $X_{0}$ may be taken to be the set of transitive points equipped with the $d_{\infty}$ metric.

Proof. Since $G$ acts transitively by isometries on $X_{0}$, it acts minimally. Thus every point $y \in X_{0}$ has orbit dense in $X_{0}$ and so $\iota(y)=: x$ has orbit dense in $X$. We now must show that $x$ is an equicontinuous point. Fix $\varepsilon>0$, and let $x^{\prime}:=\iota\left(y^{\prime}\right)$. Using the continuity of $\iota^{-1}$ and uniform continuity of $\iota$ we can choose $\delta>0$ such that if $d\left(x, x^{\prime}\right)<\delta$, then for all $g \in G, g \cdot y$ and $g . y^{\prime}$ are sufficiently close that $d\left(\iota(g . y), \iota\left(g \cdot y^{\prime}\right)\right)=d\left(g \cdot x, g, x^{\prime}\right)<\varepsilon$. In other words, $x$ is an equicontinuous point.

For the converse, let $(X, G)$ be an almost equicontinuous, transitive system and let $X_{0}$ be the set of all transitive points equipped with the $d_{\infty}$ metric. Then the inclusion $\iota: X_{0} \rightarrow X$ is a contraction and hence uniformly continuous. By Proposition 2.2, part (4), $\iota$ is a homeomorphic embedding. So, in fact, $\iota$ is a uniformly continuous $G$-compactification, as desired.

According to Proposition 2.3. constructing almost equicontinuous systems is equivalent to constructing equicontinuous compactifications of transitive isometric $G$-actions. One simple way to construct an almost equicontinuous $G$-action is to let $X_{0}=G$ with the metric $d(g, h)=1$ if and only if $g \neq h$. This is an invariant metric giving the discrete topology. One could then take the one-point compactification of $G$ and extend the left multiplication action of $G$ by fixing the point at infinity. The infinite point is sensitive and all other points are equicontinuous and transitive.

From a topological perspective, this example is not very interesting. Notice that, except for the point at infinity, none of the transitive points are recurrent; i.e., it is not true that for every transitive point $x$, every neighborhood $U \ni x$, and every compact $K \subset G$ we can find $g \notin K$ with $g . x \in U$.

If $G=\mathbb{Z}$ we can construct more examples by taking $X=X_{0}$ to be some compact monothetic group (a group with a dense cyclic subgroup). Such examples are well known and abundant. They occur precisely as Pontryagin duals of subgroups of the circle equipped with the discrete topology. Again, these examples are not very dynamically interesting because they are minimal and equicontinuous.

If we take $X_{0}$ to be a non-compact monothetic group, then we can easily pick a metric on it with respect to which $\mathbb{Z}$ acts by isometries. Then any uniform compactification $\iota: X_{0} \rightarrow X$ gives a transitive almost equicontinuous system which is not minimal and equicontinuous. In fact, it is not hard to see that any transitive 
isometric $\mathbb{Z}$-action on a complete metric space $X$ is actually a monothetic group. This is explored in detail in [2].

Now we will show how to construct an example of a $G$-system which is transitive, almost equicontinuous, and recurrent, but not minimal and equicontinuous. If one analyzes the procedure, we exploit the existence of non-compact monothetic groups. Some examples of such groups are known (see [10]) and any of them may be used in our construction. We will show a different method for constructing such groups. First we prove some obvious propositions which reduce the problem of defining isometric transitive $G$-actions to defining norms on $G$.

Definition 2.4. A symmetric norm on a group $G$ is a function $g \mapsto\|g\| \in[0, \infty)$ such that $\|g\|=0$ if and only if $g=1,\|g\|=\left\|g^{-1}\right\|$, and $\|g h\| \leq\|g\|+\|h\|$. Two norms $\|\cdot\|_{i}, i=1,2$, are uniformly equivalent if for all $\varepsilon>0$ there is a $\delta>0$ such that for any $i$ and $j,\|g\|_{i}<\delta$ implies $\|g\|_{j}<\varepsilon$.

Proposition 2.5. Transitive, isometric, free $G$-actions on complete, pointed metric spaces are in one-to-one correspondence with symmetric norms on $G$.

Proof. First, suppose $G$ acts transitively, isometrically, and freely on a complete pointed metric space $(X, x)$. Define $\|g\|=d(x, g . x)$. Since the action is free, $x \neq g . x$ unless $g=1$. Since the action is isometric, $\left\|g^{-1}\right\|=d\left(x, g^{-1} \cdot x\right)=d(g \cdot x, x)=\|g\|$. Finally

$$
\|g h\|=d(x, g h . x) \leq d(x, g \cdot x)+d(g . x, g h . x)=d(x, g . x)+d(x, h . x)=\|g\|+\|h\| .
$$

Now suppose we have a symmetric norm $\|\cdot\|$ on $G$. Then we can define a left invariant metric on $G$ by $d(g, h)=\left\|g^{-1} h\right\|$. Let $X$ be the completion of $G$ with respect to this metric and choose $x=1$ for the base point. Then $G$ obviously acts on $X$ transitively, isometrically, and freely.

A point $x$ is said to be recurrent if for every neighborhood $U \ni x$, outside every compact subset of $G$ we can find $g$ such that $g . x \in U$. A $G$-action is said to be recurrent if every point is recurrent. Given a sequence $g_{n} \in G$, we will say $g_{n}$ tends to infinity and write $g_{n} \rightarrow \infty$ if for any compact $K \subset G$, there are only finitely many $n$ with $g_{n} \in K$.

Proposition 2.6. Fix a symmetric norm $\|\cdot\|$ on $G$. The associated isometric action on the completion $X$ of $G$ is recurrent if and only if there exist $g_{n} \in G$ such that $g_{n} \rightarrow \infty$ while $\left\|g_{n}\right\| \rightarrow 0$. Furthermore $X$ is compact if and only if for all $\varepsilon>0$ the set $\{g \in G:\|g\|<\varepsilon\}$ is left syndetic.

Proof. Assume $\left\|g_{n}\right\| \rightarrow 0$. Fix any point $x \in X$ and write $x_{0}=1$ for the base point of $X$. Choose $g \in G$ with $g . x_{0} \in B_{\varepsilon}(x)$. Then

$$
\begin{aligned}
d\left(x, g g_{n} g^{-1} \cdot x\right) & \leq d\left(x, g \cdot x_{0}\right)+d\left(g \cdot x_{0}, g g_{n} \cdot x_{0}\right)+d\left(g g_{n} \cdot x_{0}, g g_{n} g^{-1} \cdot x\right) \\
& <\varepsilon+d\left(x_{0}, g_{n} \cdot x_{0}\right)+d\left(\left(g g_{n} g^{-1}\right) g \cdot x_{0},\left(g g_{n} g^{-1}\right) \cdot x\right) \\
& <\varepsilon+d\left(x_{0}, g_{n} \cdot x_{0}\right)+\varepsilon .
\end{aligned}
$$

But the middle term is equal to $\left\|g_{n}\right\|$, which tends to 0 . So $x$ is recurrent.

For the converse, we use the same argument but reverse the roles of $x$ and $x_{0}$. We assume $g_{n} . x \rightarrow x$ and $h_{n} . x \rightarrow x_{0}$. We see that we can make $h_{n} g_{n} h_{n}^{-1}$ leave every compact set, while at the same time $\left\|h_{n} g_{n} h_{n}^{-1}\right\|=d\left(x_{0}, h_{n} g_{n} h_{n}^{-1} \cdot x_{0}\right) \rightarrow 0$.

If $X$ is compact, then $(X, G)$ is a minimal equicontinuous system. So the set of return times $R$ of $x_{0}$ to $B_{\varepsilon}(x)$ is left syndetic. But $R$ is precisely $\{g \in G:\|g\|<\varepsilon\}$. 
Conversely, if this set is left syndetic, then we can choose a finite set $F \subset G$ such that $F R=G$. It follows that for every $\varepsilon>0$ and every $x \in X$ we can find $f \in F$ and $g \in R$ such that $d\left(x, f g \cdot x_{0}\right)<\varepsilon$. But

$$
d\left(x, f . x_{0}\right)<d\left(x, f g \cdot x_{0}\right)+d\left(f g \cdot x_{0}, f \cdot x_{0}\right)<\varepsilon+d\left(g \cdot x_{0}, x_{0}\right)=\varepsilon+\|g\|<2 \varepsilon .
$$

This proves that the finite set $F . x_{0}$ is $2 \varepsilon$-dense. It follows that $X$ is compact.

Suppose $\varphi$ is any symmetric nonnegative function on $G$ which takes the value 0 at 1 . Define

$$
\|h\|=\inf \left\{\varphi\left(h_{1}\right)+\cdots+\varphi\left(h_{n}\right): h_{1} \cdots h_{n}=h\right\} .
$$

Certainly $\|1\|=0$ and $\left\|h^{-1}\right\|=\|h\|$. Fix $h, h^{\prime} \in G$. Given $\varepsilon>0$ we can choose $h_{1}, \ldots, h_{n+k}$ such that $h_{1} \cdots h_{n}=h, h_{n+1} \cdots h_{n+k}=h^{\prime}$ and

$$
\varphi\left(h_{1}\right)+\cdots+\varphi\left(h_{n}\right)<\|h\|+\varepsilon, \quad \varphi\left(h_{n+1}\right)+\cdots+\varphi\left(h_{n+k}\right)<\left\|h^{\prime}\right\|+\varepsilon .
$$

Then $h h^{\prime}=h_{1} \cdots h_{n+k}$ and so

$$
\left\|h h^{\prime}\right\| \leq \varphi\left(h_{1}\right)+\cdots \varphi\left(h_{n+k}\right)<\|h\|+\left\|h^{\prime}\right\|+2 \varepsilon .
$$

It follows that $\left\|h h^{\prime}\right\| \leq\|h\|+\left\|h^{\prime}\right\|$. Except for the possibility that $\|g\|=0$ for some $g \neq 1$, the function $\|\cdot\|$ as defined above is a symmetric norm.

Write $G_{\varepsilon}$ for the subgroup generated by $\{g \in G: \varphi(g)<\varepsilon\}$. It is easy to see that if $g \notin G_{\varepsilon}$, then $\|g\| \geq \varepsilon$. So, if for each $g \in G$ with $g \neq 1$ there exists $\varepsilon>0$ such that $g \notin G_{\varepsilon}$, then $\|\cdot\|$ is a symmetric norm. This condition is far from necessary. For instance, suppose $G=\mathbb{Z}=\langle t\rangle$ acts on the circle $X=\mathbb{T}$ by an irrational rotation. Define $\|g\|$ to be the distance from 1 to $g .1$. Notice that for any $n$, the subaction generated by $t^{n}$ is also minimal. It follows that for any $\varepsilon>0$ we can find $n$ and $k$ such that $t^{n}$ and $t^{k n+1}$ both have norm less than $\varepsilon$. So $t \in G_{\varepsilon}$ and $G_{\varepsilon}=G$. Nonetheless, $\|\cdot\|$ is a symmetric norm.

Assume $G$ has an element $t$ of infinite order and define a function $\varphi$ on $G$ as follows. Let $\varphi(1)=0$. For $n \geq 0$, let $\varphi\left(t^{n !}\right)=\varphi\left(t^{-n !}\right)=(n+1)^{-1}$. Elsewhere, let $\varphi \equiv 1$. Use $\varphi$ as above to construct $\|\cdot\|$. Suppose $\|g\|<n^{-1}$ and $g \neq 1$. Then we can write $g$ as a product of elements of the form $t^{k !}$ where $k \geq n$. So $g=t^{m}$ and $m=a_{1} k_{1} !+a_{2} k_{2} !+\cdots+a_{l} k_{l}$ ! where the $k_{i} \geq n$ are distinct and each $a_{i}$ is a nonzero integer. The assumption on $\|g\|$ tells us that we can do this efficiently so that $\sum_{i}\left|a_{i}\right|\left(k_{i}+1\right)^{-1}<n^{-1}$. Put the $k_{i}$ in decreasing order and observe that $\sum_{i=2}^{l}\left|a_{i}\right|<k_{1} n^{-1}$. If $a_{1}>k_{1}+1$, then we could

(1) replace $a_{1}$ by $a_{1}-\left(k_{1}+1\right)$,

(2) introduce $k_{0}=k_{1}+1$, and

(3) introduce $a_{0}=1$.

This would give us another way of representing $m$ which reduces the value of $\sum_{i}\left|a_{i}\right|\left(k_{i}+1\right)^{-1}$. Loosely speaking, instead of taking very many large 'steps', we could have taken one even larger step.

A symmetric statement can be made if $a_{1}<-k_{1}-1$. So, let us assume $\left|a_{1}\right| \leq$ $k_{1}+1$. Then

$$
\begin{aligned}
\left|m-a_{1} k_{1} !\right| & \leq\left|a_{2}\right| k_{2} !+\cdots+\left|a_{l}\right| k_{l} ! \leq\left(k_{1}-1\right) ! \sum_{i=2}^{l}\left|a_{i}\right| \\
& \leq\left(k_{1}-1\right) ! \frac{k_{1}}{n}=\frac{k_{1} !}{n} .
\end{aligned}
$$


In particular $m$ lies in the interval $\left[k_{1} !\left(a_{1}-n^{-1}\right), k_{1} !\left(a+n^{-1}\right)\right]$. Assembling these results over all possible values of $k_{1}$ and $\left|a_{1}\right| \leq k_{1}+1$ tells us that $m$ must lie in

$$
I:=\left\{i:\left\|t^{i}\right\|<\frac{1}{n}, i>0\right\} \subseteq \bigcup_{k \geq n} \bigcup_{a=1}^{k+1}\left[k !\left(a-n^{-1}\right), k !\left(a+n^{-1}\right)\right] .
$$

When $n$ is sufficiently large, $I$ misses any given interval around 0 . Also $I$ is not syndetic. Therefore, transferring the statement about exponents to the group itself, we see that

$$
\left\{g \in G:\|g\|<\frac{1}{n}, g \neq 1\right\}=\left\{t^{i}: i \in I \text { or }-i \in I\right\}
$$

is not syndetic. Furthermore, given any element $g \in G$ with $g \neq 1$, we can choose $n$ large enough that this set does not contain $g$.

Let $X_{0}$ be the completion of $G$ with respect to the metric induced by $\|\cdot\|$. It is not necessary but illuminating to observe that this is a disjoint union of noncompact monothetic groups, one for each coset of $\langle t\rangle$. By Propositions 2.5 and 2.6. $G$ acts transitively, isometrically, freely, and recurrently on the non-compact metric space $X_{0}$. Now we may choose any uniformly continuous compactification $\iota: X_{0} \rightarrow X$ and apply Proposition 2.3 to get a transitive, recurrent, non-minimal, almost equicontinuous system $(X, G)$.

\section{INVARIANT MEASURES AND ERGODICITY}

In this section we prove Theorems 1.8 and 1.9. First, we need a lemma (which is interesting in its own right).

Lemma 3.1. Let $(X, G)$ be a system with $G$ countable, and let $\mu$ be an ergodic measure of full support. Given $A \subseteq X$ of positive measure and $x \in X$, write $R=R(x, A)=\{g \in G: g . x \in A\}$. Then for $\mu$-almost every $x \in X, R R^{-1}$ is $\Delta^{\star}$.

The author would like to thank Vitaly Bergelson for help with this proof.

Proof. Let $S$ be an infinite subset of $G$ and choose $g=g_{S}, h=h_{S} \in S$ such that $\mu\left(g^{-1} \cdot A \cap h^{-1} \cdot A\right)>0$. Let $T(g, h)$ be the full measure set $\bigcup_{k \in G}\left(k g^{-1} \cdot A \cap k h^{-1} . A\right)$. If we make such choices for every infinite subset $S$, countability of $G \times G$ tells us that we have an at most countable collection $T\left(g_{S}, h_{S}\right)$ of full measure sets. Let $Y=\bigcap_{S} T\left(g_{S}, h_{S}\right)$. Then $\mu(Y)=1$. Take $x \in Y$. Then for any infinite $S \subseteq G$ there exist $g=g_{S}, h=h_{S} \in S$ and $k \in G$ such that $k . x \in g^{-1} . A \cap h^{-1}$.A. Therefore $g k . x \in A$ and $h k . x \in A$, so $g k, h k \in R$. Finally, $R R^{-1} \ni g k(h k)^{-1}=g h^{-1} \in S S^{-1}$, so $R R^{-1} \cap S S^{-1} \neq \emptyset$. Since $S$ was an arbitrary infinite set we have shown that $R R^{-1}$ is $\Delta^{\star}$ as desired.

The proof of Theorem 1.8 was motivated by the techniques in [7].

Proof of Theorem 1.8. Suppose $(X, G)$ is not sensitive; that is, it has an equicontinuous point $x$. Fix $\varepsilon>0$ and write $\delta_{4}=\varepsilon$. Now choose $\delta_{i}, i=1,2,3$, such that:

(1) If $d(x, y)<3 \delta_{i}$, then $d(g . x, g . y)<\delta_{i+1}$ for all $g \in G$.

(2) $3 \delta_{i}<\delta_{i+1}$.

Let $A$ be the $\delta_{1}$ ball around $x$. Since $\mu$ has full support we can choose some ergodic component $\nu$ with $\nu(A)>0$. Now we can apply Lemma 3.1 to $\nu$ and $A$ to deduce the existence of a point $y \in A$ with the property that $R:=R(y, A)$ is such that $R R^{-1}$ is $\Delta^{\star}$. 
For $g \in R$ we have

$$
\begin{gathered}
d(g \cdot x, y) \leq d(g \cdot x, g \cdot y)+d(g \cdot y, y)<\delta_{2}+\delta_{1}<2 \delta_{2} . \\
\text { So, } \quad d(x, g \cdot x) \leq d(x, y)+d(y, g \cdot x)<\delta_{1}+2 \delta_{2}<3 \delta_{2} .
\end{gathered}
$$

Taking $h \in R$ we get

$$
d\left(x, h g^{-1} \cdot x\right) \leq d(x, h \cdot x)+d\left(h g^{-1} \cdot(g \cdot x), h g^{-1} \cdot x\right)<3 \delta_{2}+\delta_{3}<\varepsilon .
$$

We have proven that $R R^{-1} \subseteq R\left(x, B_{\varepsilon}(x)\right)$. Since $\varepsilon$ was arbitrary, it follows that the set of return times of $x$ to any neighborhood of itself is $\Delta^{\star}$. In particular, $R\left(x, B_{\delta_{3}}(x)\right)$ is $\Delta^{\star}$. Given $g \in R\left(x, B_{\delta_{3}}(x)\right), d\left(x, g^{-1} \cdot x\right)=d\left(g^{-1} \cdot(g \cdot x), g^{-1} \cdot x\right)<$ $\varepsilon$. It follows that $R\left(x, B_{\delta_{3}}(x)\right) \cup R\left(x, B_{\delta_{3}}(x)\right)^{-1} \subseteq R\left(x, B_{\varepsilon}(x)\right)$. In other words, $R\left(x, B_{\varepsilon}(x)\right)$ contains a symmetric $\Delta^{\star}$ and so must be (left) syndetic (as explained in the introduction). But this is equivalent to asserting that $x$ has minimal orbit closure (see for instance [6]). Since $x$ is transitive, we conclude that $(X, G)$ is minimal. By Lemma 2.1, $(X, G)$ is equicontinuous.

Now we proceed to the proof of Theorem 1.9 . The method is completely different: it relies on Proposition 2.3 and the following simple lemma. First define the function

$$
s(x)=\inf _{\varepsilon>0} \sup \left\{\operatorname{diam}\left(g \cdot B_{\varepsilon}(x)\right): g \in G\right\}=\inf \left\{d_{\infty}-\operatorname{diam}(U): x \in U \text { open }\right\} .
$$

We call $s(x)$ the sensitivity constant at $x$. Notice that $x$ is a sensitive point if and only if $s(x)>0$.

Lemma 3.2. The function $s$ defined above is upper semi-continuous and hence measurable. If $(X, G)$ admits an ergodic measure $\mu$ of full support for which the set of all sensitive points has positive measure, then the system has sensitive dependence on initial conditions.

Proof. Suppose $U$ is a neighborhood of $x$ such that $d_{\infty}$-diam $(U)<s(x)+\varepsilon$. Then for any $y \in U, s(y) \leq d_{\infty}$-diam $(U)<s(x)+\varepsilon$. Therefore $s$ is upper semi-continuous. Measurability follows.

Since $s$ is an invariant function it is constant $\mu$-almost everywhere. Since it was assumed to take positive values on a set of positive measure, it must be equal to some $c>0, \mu$-almost everywhere. Thus $s^{-1}(c)$ is a dense set. Let $U$ be any open subset of $X$. Then $U$ contains an element of $s^{-1}(c)$. By the definition of $s$ we can find $g \in G$ such that $\operatorname{diam}(g \cdot U)>c / 2$. So, $(X, G)$ is sensitive.

Proof of Theorem [1.9, Let $(X, G)$ be an almost equicontinuous system and let $\mu$ be an ergodic probability measure of full support. Then the set $X_{0}$ of equicontinuity points is an invariant set and so must have measure 1 or 0 . If it has measure 0 , then almost every point is sensitive. By Lemma 3.2, $(X, G)$ is sensitive. If $X_{0}$ has measure 1, then by Proposition 2.3 we can think of $\mu$ as a Borel measure on $\left(X_{0}, d_{\infty}\right)$.

If $\left(X_{0}, d_{\infty}\right)$ is not compact, then for some $\varepsilon>0$ we can choose a sequence $x_{1}, x_{2}, \cdots \in X_{0}$ with $d_{\infty}\left(x_{i}, x_{j}\right)>\varepsilon$ when $i \neq j$. Cover $X_{0}$ by countably many balls of $d_{\infty}$-radius $\varepsilon / 4$. One of them must have positive measure. Call it $B$ and choose $g_{n} \in G$ such that $x_{n} \in g_{n} . B$. Since $G$ acts on $\left(X_{0}, d_{\infty}\right)$ by isometries, the balls $g_{n} . B$ are disjoint. This is ludicrous, since they are all of equal, positive measure.

So, $\left(X_{0}, d_{\infty}\right)$ must be compact. Continuity of $\left(X_{0}, d_{\infty}\right) \rightarrow(X, d)$ tells us $X_{0}$ is compact as a subspace of $X$. Density tells us $X_{0}=X$. The identity is then a 
homeomorphism and $(X, G)$ is isomorphic to an isometric system; i.e., $(X, G)$ is minimal and equicontinuous.

\section{Periodic points and minimal subsystems}

In [5], Devaney suggests three properties which define the essence of chaos. According to him, a dynamical system (i.e. a continuous map $T: X \rightarrow X$ ) should be called chaotic if it

(1) is topologically transitive,

(2) has a dense set of periodic points,

(3) has sensitive dependence on initial conditions.

It was first observed in 4 that these requirements are not independent. In fact, Banks et al. proved that the first two conditions imply the third (this is the content of Theorem 1.7). In [7, Glasner and Weiss derive this as a corollary of Theorem 1.8. They also produce a remarkably simple direct proof (their Corollary 1.4). Unfortunately, it is unclear to this author how to adapt the second argument to the case of a non-abelian acting group.

Now we set out to prove Theorem 1.12 ,

Lemma 4.1. Assume $S$ is a nice generating set for the solvable group $G$. Then there is some bound $N$ such that any $g \in G$ can be written in the form $g=s_{i_{1}}^{e_{1}} s_{i_{2}}^{e_{2}} \cdots s_{i_{N}}^{e_{N}}$ where $s_{i} \in S$ and $e_{i} \in \mathbb{Z}$.

Proof. If $G$ is abelian this is obvious, so assume $G$ has higher solvability degree. Enumerate $S=\left\{s_{1}, s_{2}, \ldots s_{n}\right\}$. We know we can write $g=s_{i_{1}}^{e_{1}} s_{i_{2}}^{e_{2}} \cdots s_{i_{k}}^{e_{k}}$ for some $k$. Rearrange this word by collecting like terms and introducing commutators where necessary; that is, rearrange the word into the form

$$
g=s_{1}^{f_{1}} c_{1} s_{2}^{f_{2}} c_{2} s_{3}^{f_{3}} c_{3} \cdots c_{n-1} s_{n}^{f_{n}} c_{n}
$$

where $c_{j} \in[G, G]$. By induction on the degree of solvability, there is some constant $M$ such that each $c_{i}$ contributes at most $M$ terms of the form $s_{a}^{b}$. So, $g$ can be written with no more than $n M+n=|S|(M+1)$ terms.

Lemma 4.2. Suppose $x$ is an equicontinuous point in a topological dynamical system $(X, G)$. If $x$ is a limit of minimal points $x_{n}$, then $x$ is minimal.

This lemma appears in [1].

Proof. Fix $\varepsilon>0$ and choose $\delta>0$ smaller than $\varepsilon / 2$ such that $g . B_{\delta}(x) \subseteq B_{\varepsilon / 2}(g \cdot x)$. Choose $y:=y_{n} \in B_{\delta}(x)$ and let $R=\left\{g \in G: g . y \in B_{\varepsilon / 2}(x)\right\}$. Since $y_{n}$ is minimal, $R$ is syndetic. If $g \in R$, then $d(g . x, x) \leq d(g \cdot x, g \cdot y)+d(g \cdot y, x) \leq \varepsilon / 2+\varepsilon / 2$. So $R \subseteq\left\{g \in G: g . x \in B_{\varepsilon}(x)\right\}$, which proves the latter set is left syndetic. Thus, $x$ is minimal.

Proof of Theorem 1.12. Suppose the system is not sensitive. Then it has an equicontinuous point. By Theorem [1.6 any transitive point must be equicontinuous. Let $x$ be such a point. Fix $y \in X=\overline{G . x}$. Choose a sequence $g_{n} \in G$ such that $g_{n} . x \rightarrow y$. Lemma 4.1 tells us that each $g_{n}$ can be written with at most $N$ terms of the form $s^{e}$. By passing to a subsequence we can find a sequence $s_{1}, s_{2}, \ldots, s_{k} \in S$ such that each $g_{n}$ is of the form $g_{n}=s_{k}^{e_{n, k}} s_{k-1}^{e_{n, k-1}} \cdots s_{1}^{e_{n, 1}}$ (we allow the possibility that $s_{i}=s_{j}$ for unequal $i, j$ ). 
Let $X_{1}$ be the orbit closure of $x$ under $\left\langle s_{1}\right\rangle$. Pass to a subsequence along which $s_{1}^{e_{n, 1}} . x$ converges to some point $x_{1} \in X_{1}$. The points which are minimal under the action of $\left\langle s_{1}\right\rangle$ are dense by assumption. So, by Lemma 4.2, $x$ is also minimal under this action. Thus we can find powers of $s_{1}$ which move $x_{1}$ arbitrarily close to $x$. Applying Lemma 2.1 we see that $x_{1}$ is another transitive equicontinuous point. Since $x$ is an equicontinuous point, as $d\left(s_{1}^{e_{n, 1}} . x, x_{1}\right) \rightarrow 0$ we also have

$$
d\left(s_{k}^{e_{n, k}} \cdots s_{2}^{e_{n, 2}} \cdot\left(s_{1}^{e_{n, 1}} \cdot x\right), s_{k}^{e_{n, k}} \cdots s_{2}^{e_{n, 2}} \cdot x_{1}\right) \rightarrow 0
$$

This proves that $s_{k}^{e_{n, k}} \cdots s_{2}^{e_{n, 2}} \cdot x_{1} \rightarrow y$.

Repeat this process: let $X_{2}$ be the orbit closure of $x_{1}$ under $\left\langle s_{2}\right\rangle$. Pass to a subsequence along which $s_{2}^{e_{n, 2}} \cdot x_{1}$ converges to some point $x_{2} \in X_{2}$. Argue as above to conclude that $X_{2}$ is minimal under the action of $\left\langle s_{2}\right\rangle$ and that $x_{2}$ is transitive equicontinuous and satisfies $s_{k}^{e_{n, k}} \cdots s_{3}^{e_{n, 3}} \cdot x_{2} \rightarrow y$.

Continuing in this way we get a transitive equicontinuous point $x_{k-1}$, whose orbit closure $X_{k}$ under the action of $s_{k}$ is minimal and contains $y$. Then we can find some powers of $s_{k}$ which move $y$ as close as we like to $x_{k-1}$. By Lemma 2.1, $y$ is a transitive, equicontinuous point.

We have shown that every point is transitive and equicontinuous. Equivalently, $(X, G)$ is a minimal equicontinuous system.

Corollary 4.3. Let $G$ and $S$ be as in Theorem 1.12 and assume that the set of periodic points for $(X,\langle s\rangle)$ is dense for each $s \in S$ (where $\langle s\rangle$ is the group generated by $s)$. If $(X, G)$ is not sensitive, then $X$ must be a finite set on which $G$ acts transitively.

It would be advantageous to drop the condition that $S$ be nice in Theorem 1.12 . Unfortunately, the following example demonstrates that this condition (or something like it) is unavoidable. Let $G$ be the solvable group $\mathbb{Z} \rtimes \mathbb{Z} / 2 \mathbb{Z}$ and let $a$ and $b$ be the generators of the factors $\mathbb{Z}$ and $\mathbb{Z} / 2 \mathbb{Z}$ respectively. Then $S:=\{a, a b\}$ is another generating set for $G$. Let $X$ be the one point compactification of $G$ and extend the left multiplication action of $G$ on itself to an action on $X$ by fixing the point at infinity. The system $(X, G)$ is transitive but not minimal.

Each $s \in S$ has order two. So, every point is minimal for the system $(X,\langle s\rangle)$. However, $(X, G)$ does not have sensitive dependence on initial conditions. In fact, the only sensitive point is the point at infinity. All other points are isolated and therefore equicontinuous. This is not a counterexample to Theorem 1.12 because $S$ is not nice. Suppose we add more generators to $S$ to make it nice. For instance we could take $S=\{a, a b,[a, a b]\}$. Then the hypotheses of the theorem are not met: for any $x \in X, \lim _{|n| \rightarrow \infty}[a, a b]^{n} . x=\infty$, which proves that the only minimal point of $(X,\langle[a, a b]\rangle)$ is $\infty$ (clearly not dense).

\section{ACKNOWLEDGEMENTS}

The author would like to thank Vitaly Bergelson for proposing this avenue of research, Cory Christopherson for proofreading an early version, and Michael Hochman for making him aware of [1. The author would especially like to thank Rafal Pikula for thoroughly checking the final draft. 


\section{REFERENCES}

1. E. Akin, J. Auslander, and K. Berg. When is a transitive map chaotic? In Convergence in ergodic theory and probability, Ohio State Univ. Math. Res. Inst. Publ., 5, pages 25-40. de Grutyer, Berlin, 1996. MR1412595 (97i:58106)

2. E. Akin and E. Glasner. Residual properties and almost equicontinuity. J. Anal. Math. 84: 243-286, 2001. MR 1849204 (2002f:37020)

3. J. Auslander and J. Yorke. Interval maps, factors of maps, and chaos. Tôhoku Math J. 32(2): 177-188, 1980. MR.580273 (82b:58049)

4. J. Banks, J. Brooks, G. Cairns, G. Davis, and P. Stacey. On Devaney's definition of chaos. Math. Monthly 99(4): 332-334, 1992. MR1157223 (93d:54059)

5. R. L. Devaney. An introduction to chaotic dynamical systems. Addison-Wesley, Redwood City, CA, 1989. MR1046376 (91a:58114)

6. E. Glasner. Ergodic theory via joinings. Mathematical Surveys and Monographs, 101. American Mathematical Society, Providence, RI, 2003. MR1958753 (2004c:37011)

7. E. Glasner and B. Weiss. Sensitive dependence on initial conditions. Nonlinearity 6(6): 10671075, 1993. MR 1251259 (94j:58109)

8. J. Guckenheimer. Sensitive dependence on initial conditions for one-dimensional maps. Comm. Math. Phys. 70(2): 133-160, 1979. MR.553966 (82c:58037)

9. E. Kontorovich and M. Megrelishvili. A note on sensitivity of semigroup actions. Semigroup Forum 76(1): 133-141, 2008. MR2367162(2008j:37027)

10. S. Rolewicz. Some remarks on monothetic groups. Colloq. Math. 13: 27-28, 1964. MR0171876 (30:2102)

11. P. Walters. An introduction to ergodic theory. Graduate Texts in Mathematics, 79. SpringerVerlag, New York-Berlin, 1982. MR648108 (84e:28017)

Department of Mathematics, The Ohio State University, 231 W. 18th Avenue, ColumBus, OHIо 43210

E-mail address: polof@math.osu.edu 